\title{
$S$-PLANE ANALYSIS FOR THE FUNDAMENTAL PROBLEMS OF A STRETCHED INFINITE PLATE WEAKENED BY CURVILINEAR HOLES
}

\author{
I. S. ISMAIL
}

Received 29 April 2002

\begin{abstract}
Complex variable methods are used to obtain exact and closed expressions for Goursat's functions for the stretched infinite plate weakened by two inner holes which are free from stresses. The plate considered is conformally mapped on the area of the right half-plane. Previous work is considered as special cases of this work. Cases of different shapes of holes are included. Also, many new cases are discussed using this mapping.
\end{abstract}

2000 Mathematics Subject Classification: 74B99, 74K20, 74G99.

1. Introduction. The boundary value problems for isotropic homogeneous perforated infinite plates have been discussed by several authors [1, 2, 3, 4, 5, 6, 7, 8, 9]. Muskhelishvili [9] solved the problem of stretching an infinite plate weakened by an elliptic hole by using the transformation

$$
Z=c\left(\xi+m \xi^{-1}\right)
$$

This transformation conformally maps the infinite domain bounded internally by an elliptic onto the domain outside the unit circle $|\xi|=1$ in the $\xi$-plane.

Muskhelishvili also used the Hilbert problem to discuss the case of a stretched infinite plate weakened by a circular cut.

He showed also that the first and second fundamental problems in the plane theory of elasticity are equivalent to finding two analytic functions $\phi_{1}(z)$ and $\psi_{1}(z)$ of one complex argument $z=x+i y$ satisfying the boundary conditions

$$
k \phi_{1}(t)-t \overline{\phi_{1}^{\prime}(t)}-\overline{\psi_{1}(t)}=f(t),
$$

where $k=-1, f(t)$ is a given function of stresses for the first fundamental problem, while $k=x=(\lambda+3 \mu) /(\lambda+\mu)>1, f=2 \mu g(t)$ is a given function of the displacement for the second fundamentals. $\lambda, \mu$ are called the Lame's constants, $\chi$ is called Muskhelishvili's constant, and $t$ denotes the affix of a point of the boundary $L$.

In [5], El-Sirafy used the complex variable methods and rational mapping function to obtain the Goursat functions for a stretched infinite plate weakened by an inner curvilinear hole $\Gamma$, conformally mapped on the right half-plane (Res $\geq 0$ ) by the transformation

$$
z=c w(\xi)=c \frac{(s+1)^{2}+m(s-1)^{2}}{s^{2}-1-n(s-1)^{2}}, \quad c>0,|n|<1, s=\sigma+i \tau .
$$


The same author in [6] considered the case of stretched infinite plate weakened by hypotrochoidal holes. The two functions $\phi(z)$ and $\psi(z)$ were obtained in closed form. In [1], Abdou and Hassan obtained the two Gourstat's functions for the stretched infinite plate weakened by a hole whose edge is free from stresses, using the two rational mapping functions

$$
z=c \frac{\xi+m \xi^{-2}}{1-n \xi^{-1}}, \quad z=c \frac{\xi+m \xi^{-2}}{1-n \xi^{-2}}
$$

where $m, n$ are real parameters subject to the conditions that $z^{\prime}(\xi) \neq 0$ or $\infty$ for $|\xi|>1$. In this paper, Cauchy integral methods and rational mapping function

$$
\begin{aligned}
& z=c w(s)=c \frac{(s+1)\left[(s+1)^{2}+m(s-1)^{2}\right]}{(s-1)\left[(s+1)-n_{1}(s-1)\right]\left[(s+1)-n_{2}(s-1)\right]}, \\
& c>0, \quad\left|n_{1}\right|<1, \quad\left|n_{2}\right|<1, \quad \operatorname{Re} s \geq 0, \quad s=\sigma+i \tau, \quad n_{1} \neq n_{2},
\end{aligned}
$$

are used to obtain exact and closed expressions for Goursat functions for the first and second fundamental problems of an infinite plate weakened by curvilinear holes conformally mapped on the domain onto the right half-plane.

$m, n_{1}$, and $n_{2}$ are real parameters subject to the conditions that $w(\infty)$ is bounded and $w^{\prime}(s)$ does not vanish in the right half-plane (i.e., $\operatorname{Re} s \geq 0$ ). Some applications of the first and second fundamental problems on these domains are investigated. The interesting cases of an infinite plate weakened by an elliptic hole, a crescent-like hole, or a cut having the shape of a circular arc, and the hypotrochoidal hole with three rounded corners are considered as special cases. Functions $\phi(z)$ and $\psi(z)$ are obtained in closed form.

2. Mapping function. The mapping function given in (1.5) maps the area outside curvilinear contours in the $z$-plane in the half-plane $\operatorname{Re} s \geq 0$, where the constants $m$, $n_{1}$, and $n_{2}$ are real parameters subject to the conditions that $w(\infty)$ is bounded and $w^{\prime}(s)$ does not vanish in the right half-plane.

In (1.5), if we let $s=(\xi+1) /(\xi-1)$, we have the transformation mapping

$$
z=c w(\xi)=c \frac{\xi+m \xi^{-1}}{\left(1-n_{1} \xi^{-1}\right)\left(1-n_{2} \xi^{-1}\right)} .
$$

Here, the mapping function maps the area outside curvilinear holes in the $z$-plane on the area outside the unit circle $\gamma$ in the $\xi$-plane under the condition $z^{\prime}(\xi) \neq 0$ or $\infty$ for $|\xi|>1$.

Assuming that $\tau$ is the parameter of the curve, the parametric equations of (1.5) are

$$
\begin{gathered}
x=\frac{c}{g(\tau)}\left(\ell_{1} M_{1}+\ell_{2} M_{2}\right), \\
y=\frac{c}{g(\tau)}\left(\ell_{2} M_{1}+\ell_{1} M_{2}\right), \\
g(\tau)=M_{1}^{2}+M_{2}^{2},
\end{gathered}
$$




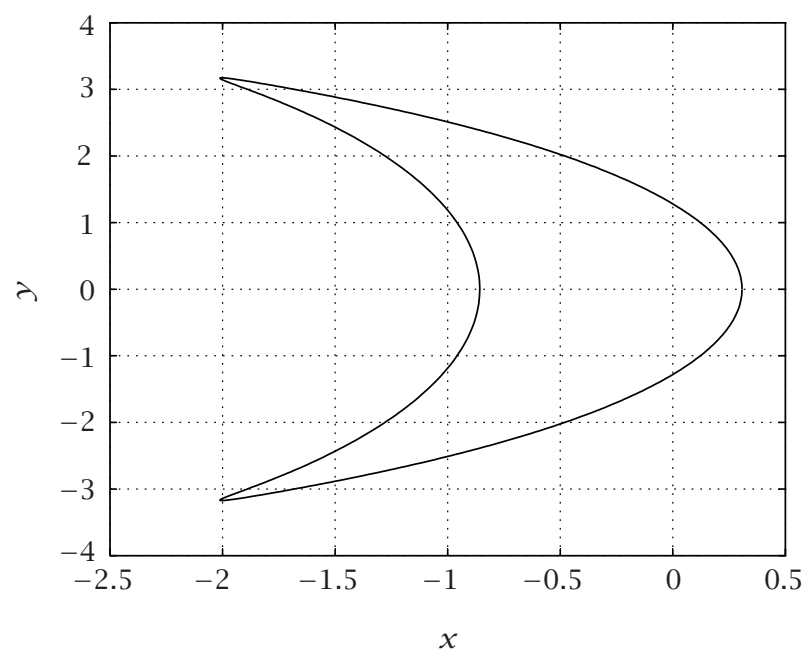

FIGURE 2.1. Plotting of parametric equation of the mapping $z=2(\xi-$ $\left.0.76 \xi^{-1}\right) /\left(1+0.3 \xi^{-1}\right)\left(1+0.2 \xi^{-1}\right)$.

where

$$
\begin{aligned}
\ell_{1} & =(1+m)+(m-2) \tau^{2}, \quad \ell_{2}=\left[3-m-(1+m) \tau^{2}\right] \tau, \\
M_{1} & =-\left(1+n_{1}+n_{2}+n_{1} n_{2}\right)+\left(n_{2}-n_{1}+3 n_{1} n_{2}-1\right) \tau^{2}, \\
M_{2} & =\left[s n_{1} n_{2}-\left(1+n_{1}+n_{2}+n_{1} n_{2}\right) \tau^{2}-n_{2}-n_{1}-1\right] \tau .
\end{aligned}
$$

The shapes of the curvilinear holes depend on the parameters in (2.2), (2.3). Various shapes with several axes of symmetery can be obtained (see Figures 2.1, 2.2, 2.3, and 2.4).

3. Basic equations. Consider a region of an elastic media of an infinite plate denoted by $S$ and bounded by a single contour $L$, with a curvilinear hole.

If $\overline{x x}, \overline{y y}$, and $\overline{x y}$ represent the components of stress, while $u, v$ represent those of displacement, in the absence of body forces, the formula of Kolosov-Muskhelishvili [9] has the following form:

$$
\begin{gathered}
\overline{x x}+\overline{y y}=4 \operatorname{Re}\left[\phi_{1}^{\prime}(z)\right], \\
\overline{y y}-\overline{x x}+2 i \overline{x y}=2\left[\bar{z} \phi_{1}^{\prime \prime}(z)+\psi^{\prime}(z)\right], \\
2 \mu(u+i v)=k \phi_{1}(z)-z \overline{\phi_{1}^{\prime}(z)}-\overline{\psi_{1}(z)} .
\end{gathered}
$$

In terms of the conformal mapping function,

$$
z=c w(\xi), \quad c>0, \quad w^{\prime}(\xi) \neq 0 \text { or } \infty \quad \text { for }|\xi|>1 .
$$

The infinite region outside a closed contour is conformally mapped on the region outside the unit circle $\gamma$. 


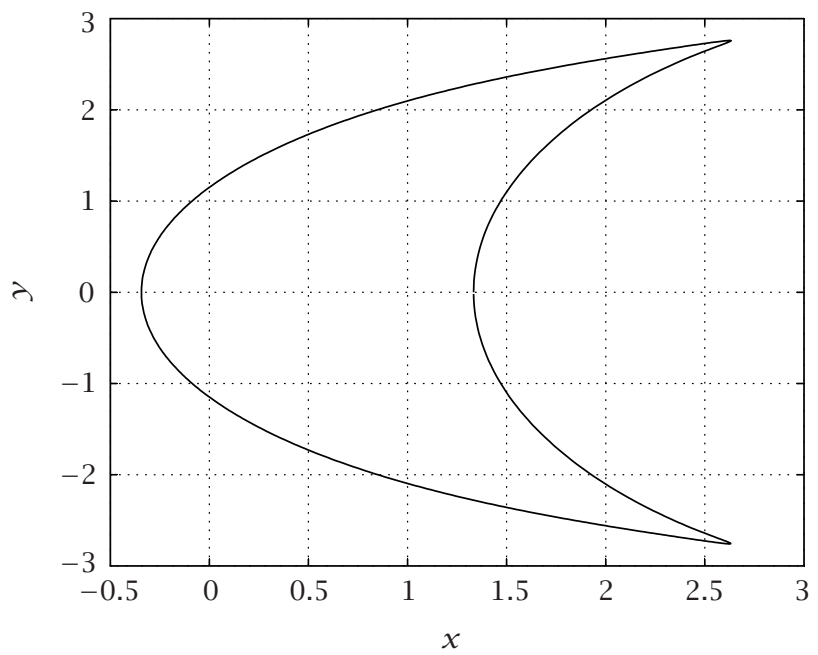

FIGURE 2.2. Plotting of parametric equation of the mapping $z=2(\xi-$ $\left.0.7 \xi^{-1}\right) /\left(1-0.25 \xi^{-1}\right)\left(1-0.4 \xi^{-1}\right)$.

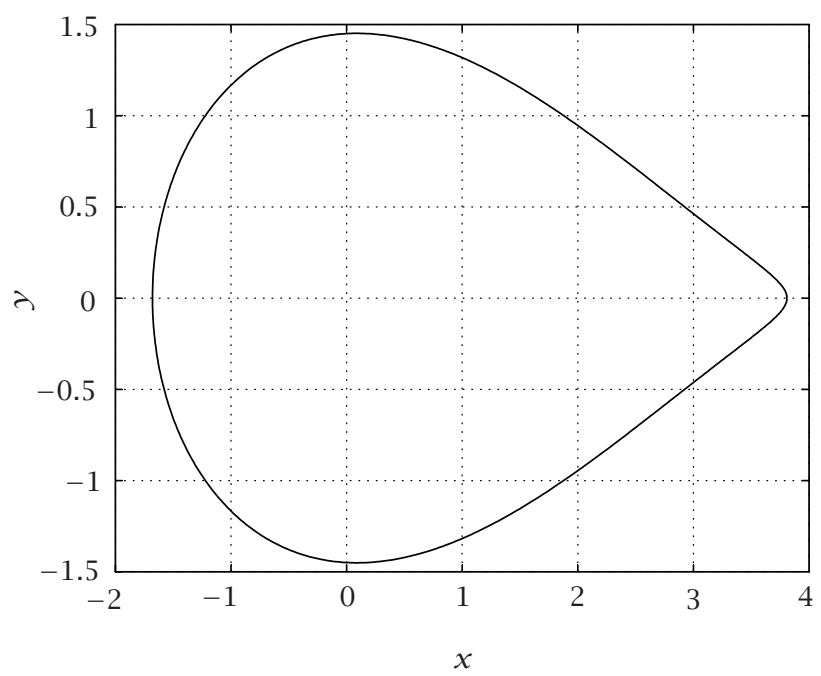

FIGURE 2.3. Plotting of parametric equation of the mapping $z=2(\xi+$ $\left.0.2 \xi^{-1}\right) /\left(1-0.1 \xi^{-1}\right)\left(1-0.3 \xi^{-1}\right)$.

The complex potentials $\phi_{1}(z)$ and $\psi_{1}(z)$ can be written in the form

$$
\begin{aligned}
& \phi_{1}(t)=-\frac{X+i Y}{2 \pi(1+\chi)} \ell n t+c \Gamma t+\phi(t), \\
& \psi_{1}(t)=\frac{\chi(X+i Y)}{2 \pi(1+\chi)} \ell n t+c \Gamma^{*} t+\psi(t),
\end{aligned}
$$




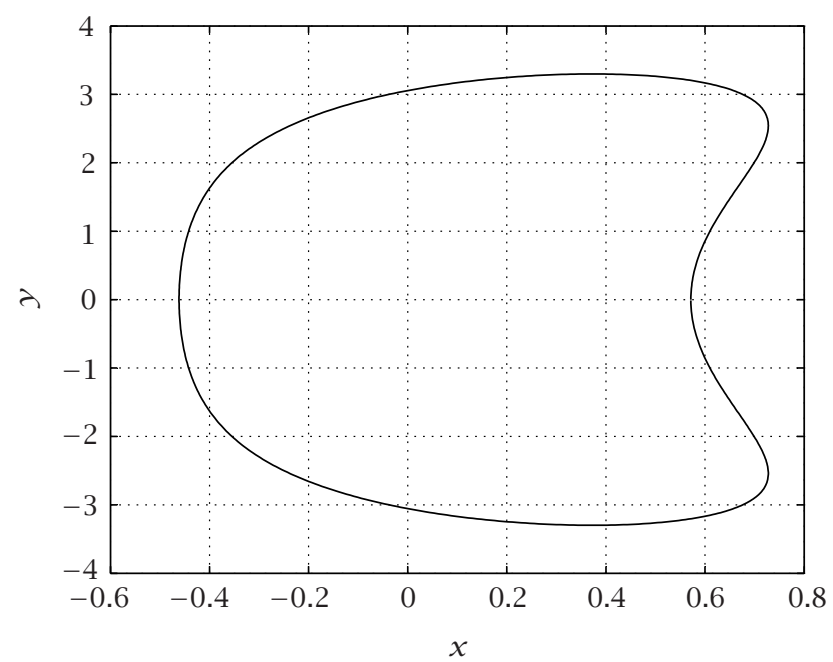

FIGURE 2.4. Plotting of parametric equation of the mapping $z=2(\xi-$ $\left.0.76 \xi^{-1}\right) /\left(1-0.3 \xi^{-1}\right)\left(1+0.2 \xi^{-1}\right)$.

where $x, y$ are the components of the resultant vector of all external forces acting on single contour $L$ while $\Gamma, \Gamma^{*}$ are complex constants.

Generally, functions $\phi(\xi)$ and $\psi(\xi)$ are single-valued analytic functions within the region outside the unit circle, and $\phi(\infty)=0$.

Substituting (3.4) in (3.2), we obtain

$$
k \phi(t)-t \overline{\phi^{\prime}(t)}-\overline{\psi(t)}=f(t),
$$

where $k=-1, f(t)=-f^{*}(t)$ for the first displacement, while $k=\chi, f(t)=2 \mu g(t)$ for the second fundamental problem.

4. Method of solution. Since

$$
\frac{\overline{w(i \tau)}}{\overline{w^{\prime}(i \tau)}}=\overline{\alpha(\tau)}+\beta(i \tau)
$$

where

$$
\begin{gathered}
\alpha(\tau)=\sum_{j=1}^{2} \frac{h_{j}}{a_{j}+i \tau}, \quad a_{j}=\frac{1+n_{j}}{1-n_{j}} \quad(j=1,2), \\
h_{1}=\frac{\lambda_{1}\left(a_{1}, a_{2}\right)}{J_{01}}, \quad h_{2}=\frac{\lambda_{1}\left(a_{2}, a_{1}\right)}{J_{02}}, \\
J_{01}=\left(a_{1}+1\right)\left(a_{1}-a_{2}\right)\left[\lambda_{2}\left(a_{1}, a_{2}\right)-\lambda_{3}\left(a_{1}, a_{2}\right)\right], \\
J_{02}=\left(a_{2}+1\right)\left(a_{2}-a_{1}\right)\left[\lambda_{2}\left(a_{2}, a_{1}\right)-\lambda_{3}\left(a_{2}, a_{1}\right)\right], \\
\lambda_{1}\left(a_{1}, a_{2}\right)=4 a_{1}^{2}\left(a_{1}-1\right)^{3}\left(a_{1}+a_{2}\right)^{2}\left[\left(a_{1}-1\right)^{2}+m\left(a_{1}+1\right)^{2}\right], \\
\lambda_{2}\left(a_{1}, a_{2}\right)=2 a_{1}\left(a_{1}-1\right)\left(a_{1}+a_{2}\right)\left[3\left(a_{1}+1\right)^{2}+2 m\left(a_{1}^{2}-1\right)+m\left(a_{1}-1\right)^{2}\right], \\
\lambda_{3}\left(a_{1}, a_{2}\right)=\left(a_{1}+1\right)\left[\left(a_{1}+1\right)^{2}+m\left(a_{1}-1\right)^{2}\right]\left[2 a_{1}\left(a_{1}-1\right)+\left(a_{1}+a_{2}\right)\left(3 a_{1}-1\right)\right],
\end{gathered}
$$


and

$$
\beta(s)=\frac{1}{\left(s-a_{1}\right)\left(s+a_{2}\right)}\left[\frac{H(s)}{E(s)}+h_{1}\left(s-a_{2}\right)+h_{2}\left(s-a_{1}\right)\right] .
$$

Notice that $\beta(s)$ is a regular function within the right half-plane except at infinity, where

$$
\begin{aligned}
H(s)=(s-1)^{3}\left[(s-1)^{2}+m(s+1)^{2}\right]\left(s+a_{1}\right)^{2}\left(s+a_{2}\right)^{2}, \\
E(s)=(s+1)\left\{\left[(s-1)\left(s+a_{1}\right)\left(s+a_{2}\right)\right]\left\lfloor 3(s+1)^{2}+2 m\left(s^{2}-1\right)+m(s-1)^{2}\right\rfloor\right. \\
\left.-\left[(s+1)^{3}+m(s+1)(s-1)^{2}\right]\left[(s-1)\left(2 s+a_{1}+a_{2}\right)+\left(s+a_{1}\right)\left(s+a_{2}\right)\right]\right\} .
\end{aligned}
$$

The boundary condition (3.5) takes the form

$$
k \phi(i \tau)-\alpha(\tau) \overline{\phi^{\prime}(i \tau)}-\overline{\psi(i \tau)}=f_{*}(\tau),
$$

where

$$
\begin{aligned}
\phi(s)= & \phi(w(s)), \\
f_{*}(\tau)= & f(w(i \tau))-\sum_{j=1}^{2} \gamma_{0 j}+w(i \tau)(\bar{\Gamma}-k \Gamma)+\overline{w(i \tau)} \overline{\Gamma^{*}} \\
& -\frac{X-i Y}{2 \pi(1+\chi) \overline{w^{\prime}(i \tau)}}\left[w(i \tau)-\overline{w^{\prime}(i \tau)}\right] \quad(j=1,2), \\
\psi(s)= & \psi(w(s))+\beta(s) \phi^{\prime}(s)+\sum_{j=1}^{2} \gamma_{0 j}-\frac{X+i Y}{2 \pi(1+\chi)}, \\
\gamma_{0 j}= & c\left(\frac{1+m}{1-n_{j}}\right)\left(\bar{\Gamma}-k \Gamma+\overline{\Gamma^{*}}\right) \quad(j=1,2) .
\end{aligned}
$$

Assuming $\phi(\infty)=\psi(\infty)=0$, then multiplying both sides of (4.5) by $1 / 2 \pi(s-i \tau)$ and integrating with respect to $\tau$ from $\infty$ to $-\infty$, we have

$$
k(\phi)(s)-\frac{1}{2 \pi} \int_{-\infty}^{\infty} \frac{\alpha(\tau) \overline{\phi^{\prime}(i \tau)}}{s-i \tau} d \tau=\frac{1}{2 \pi} \int_{-\infty}^{\infty} \frac{f_{*}(\tau)}{S-i \tau} d \tau .
$$

Substituting (4.1) in (4.7), we obtain

$$
\begin{gathered}
\frac{1}{2 \pi} \int_{-\infty}^{\infty} \frac{\alpha(\tau) \overline{\phi^{\prime}(i \tau)}}{s-i \tau} d \tau=\sum_{j=1}^{2} \frac{c b_{j} h_{j}}{s+a_{j}} \\
\frac{1}{2 \pi} \int_{-\infty}^{\infty} \frac{f_{*}(\tau) d \tau}{s-i \tau}=f_{1}(s)-\frac{2 c \overline{\Gamma^{*}}}{1+s}+2 c(k \Gamma-\bar{\Gamma}) \sum_{j=1}^{2} \frac{m+n_{j}^{2}}{\left(1-n_{j}^{2}\right)\left(s+a_{j}\right)} \\
+\frac{X-i Y}{1+X} \sum_{j=1}^{2} \frac{n_{j} a_{j}\left(m+n_{j}^{2}\right)}{\left(1+m n_{j}^{2}\right)\left(s+a_{j}\right)} \\
A_{1}(s)=\frac{1}{2 \pi} \int_{-\infty}^{\infty} \frac{f(w(i \tau))}{s-i \tau} d \tau,
\end{gathered}
$$


where $b_{j}(j=1,2)$ are complex constants to be determined. Substituting from (4.8), (4.9), and (4.10) in (4.7), we get

$$
\begin{aligned}
k \phi(s)= & A_{1}(s)+\frac{2 c \overline{\Gamma^{*}}}{1+s} \\
& +\sum_{j=1}^{2}\left[\frac{c h_{j} b_{j}}{s+a_{j}}+\frac{2 c(k \Gamma-\bar{\Gamma})}{\left(1-n_{j}\right)^{2}}\left(\frac{m+n_{j}^{2}}{s+a_{j}}\right)+\frac{n_{j} a_{j}\left(m+n_{j}^{2}\right)(X-i Y)}{\pi\left(1+m n_{j}^{2}\right)(1+\chi)\left(s+a_{j}\right)}\right] .
\end{aligned}
$$

Differentiating (4.11) and inserting $\phi(\tau)$ in (4.8), the complex constants $b_{j}$ can be determined in the form

$$
\begin{aligned}
b_{j}= & \frac{2 a_{j}^{2}}{c\left(16 a_{j}^{4}+h_{j}^{2}\right)}\left\{\left[8 a_{j}^{2} k \overline{A_{1}^{\prime}\left(a_{j}\right)}-2 h_{j} A_{1}^{\prime}\left(a_{j}\right)\right]+c\left(1-n_{j}\right)^{2}\left(4 a_{j}^{2} k-h_{g} \overline{\Gamma^{*}}\right)\right. \\
& \left.-c\left[\left(4 a_{j}^{2} k^{2}+h_{j}\right) \bar{\Gamma}-k \Gamma\left(4 a_{j}^{2}+h_{j}\right)\right]\left[\frac{m+n_{j}^{2}}{\left(1+n_{j}\right)^{2}}\right]\right\} \\
& -\frac{n_{j}\left(m+n_{j}^{2}\right) a_{j}}{c(1+\chi)\left(1+m n_{j}^{2}\right)}\left[\frac{X}{4 a_{j}^{2} k+h_{j}}+i \frac{Y}{4 a_{j}^{2} k+h_{j}}\right] \quad(j=1,2) .
\end{aligned}
$$

Substituting (4.12) in (4.11), the function $\phi(s)$ becomes

$$
\begin{aligned}
k \phi(s)= & A_{1}(s)+\frac{2 c \overline{\Gamma^{*}}}{1+s}+\frac{k}{\pi(1+\chi)} \sum_{j=1}^{2} \frac{n_{j}\left(m+n_{j}^{2}\right) a_{j} J_{0 j}}{s+a_{j}}\left(X J_{1 j}-i Y J_{2 j}\right) \\
& +2 c(k \Gamma-\bar{\Gamma}) \sum_{j=1}^{2} \frac{\left(m+n_{j}^{2}\right)}{\left(s+a_{j}\right)\left(1-n_{j}\right)^{2}}+\sum_{j=1}^{2} \frac{2 n_{j}^{2}\left(m+n_{j}^{2}\right) J_{1 j} J_{2 j} L_{j}}{\left(1-n_{j}\right)^{2}\left(s+a_{j}\right)} .
\end{aligned}
$$

Hence from the boundary condition (3.4), we have

$$
\begin{aligned}
\psi(s)= & A_{2}(s)+B_{1}(s)+\frac{2 c(\Gamma-k \bar{\Gamma})}{1+s}+2 c \Gamma^{*} \sum_{j=1}^{2} \frac{m+n_{j}^{2}}{\left(s+a_{j}\right)\left(1-n_{j}\right)^{2}} \\
& -\frac{\overline{\Gamma^{*}}}{(1+s)^{2}} \sum_{j=1}^{2}\left(1-n_{j}\right)\left(s+a_{j}+2\right)+\sum_{j=1}^{2} \frac{n_{j}^{2}\left(s+3 a_{j}\right)\left(m+n_{j}^{2}\right)^{2}}{\left(s+a_{j}\right)^{2}} \\
& *\left\{\frac{2 c(k \Gamma-\bar{\Gamma})}{\left(1-n_{j}\right)^{2}} J_{0 j}^{-1}+\frac{a_{j}\left(X J_{1 j}-i Y J_{2 j}\right)}{\pi(1+\chi)\left(1+m n_{j}^{2}\right)}+\frac{2 n_{j}^{2} J_{1 j} J_{2 j}}{k\left(1-n_{j}\right)^{2} J_{0 j}} L_{j}\right\},
\end{aligned}
$$


where

$$
\begin{aligned}
J_{1 j}= & {\left[k J_{0 j}+n_{j}^{2}\left(m+n_{j}^{2}\right)\right]^{-1}, } \\
J_{2 j}= & {\left[k J_{0 j}-n_{j}^{2}\left(m+n_{j}^{2}\right)\right]^{-1}, } \\
L_{j}= & k\left\lfloor 2\left(1+n_{j}\right)^{2} \overline{A_{1}^{\prime}\left(a_{j}\right)}+c\left(1-n_{j}^{2}\right)^{2} \Gamma^{*}+c\left(m+n_{j}^{2}\right)(\Gamma-k \bar{\Gamma})\right\rfloor J_{0 j} \\
& -n_{j}^{2}\left(m+n_{j}^{2}\right)\left[2\left(1+n_{j}\right)^{2} A_{1}^{\prime}\left(a_{j}\right)+c\left(1-n_{j}^{2}\right)^{2} \overline{\Gamma^{*}}+c\left(m+n_{j}^{2}\right)(\Gamma-k \bar{\Gamma})\right], \\
B_{1}(s)= & \frac{1}{2 \pi} \int_{-\infty}^{\infty} \frac{\overline{A_{1}(i \tau)}}{\left(i \tau-a_{j}\right)(s-i \tau)} d \tau, \\
A_{2}= & \frac{1}{2 \pi} \int_{-\infty}^{\infty} \frac{\frac{f(w(i \tau))}{s-i \tau}}{s-i, 2) .}
\end{aligned}
$$

5. Special cases. We are now in a position to consider several interesting cases.

(A) Let $n_{2}=0$ in the transformation mapping of (2.1) and $X=Y=f=0, k=-1$, $\Gamma=P / 4$, and $\Gamma^{*}=-(P / 2) e^{-2 i \theta}$. We have firstly the transformation mapping

$$
Z=c w(s)=c \frac{(1+s)^{2}+m(s-1)^{2}}{s^{2}-1-n(s-1)^{2}}
$$

which leads to the Goursat's functions

$$
\begin{aligned}
\phi(s)= & \frac{c p}{(1-n)^{2}}\left[\frac{\left(m+n^{2}\right) J}{s+a}-\frac{(1-n)^{2} e^{2 i \theta}}{1+s}\right], \\
\psi(s)= & c P\left[\frac{1}{1+s}-\frac{\left(m+n^{2}\right) e^{2 i \theta}}{(1-n)^{2}(s+a)}\right] \\
& +c p h\left[\frac{\left(m+n^{2}\right)(s+3 a)}{4(1+n)^{2}(s+a)^{2}} J-\frac{s+2+a}{(1+a)^{2}(1+s)^{2}}\right],
\end{aligned}
$$

where

$$
J=\frac{(m+2) n^{2}-1+n^{2}\left(n^{2}-1\right) \cos 2 \theta}{n^{4}-1+2 n^{2}(1+m)}+i n^{2} \sin 2 \theta
$$

and $p$ is a uniform tensile stress.

These results are in agreement with the work of El-Sirafy [5].

(B) Let $n_{2}=0, n_{1}=n$, and $s=(\xi+1) /(\xi-1)$. We have the transformation mapping

$$
z=c w(\xi)=c \frac{\xi+m \xi^{-1}}{1-n \xi^{-1}}
$$

and the two Goursat's functions

$$
\begin{aligned}
k \phi(\xi) & =\frac{h}{n-\xi}\left(N+\frac{k E-h v \bar{E}}{k^{2}-h^{2} v^{2}}\right)-\frac{c \overline{\Gamma^{*}}}{\xi}+A(\xi), \\
\psi(\xi) & =\frac{c K \bar{\Gamma}}{\xi}-\frac{w\left(\xi^{-1}\right)}{w^{\prime}(\xi)} \phi_{*}(\xi)+\frac{h \xi}{1-n \xi} \phi_{*}\left(n^{-1}\right)+B(\xi)-B,
\end{aligned}
$$


where

$$
\begin{aligned}
& \phi_{*}(\xi)=\phi^{\prime}(\xi)+c \Gamma-\frac{X+i Y}{2 \pi(1+\chi) \xi}, \\
& N=c \bar{\Gamma}-\frac{X-i Y}{2 \pi(1+\chi)} n, \\
& h=\frac{\left(m+n^{2}\right)\left(1-n^{2}\right)^{2}}{1-(m+2) n^{2}}, \\
& v=\frac{n^{2}}{\left(1-n^{2}\right)^{2}}, \\
& E=-\overline{A^{\prime}\left(n^{-1}\right)}-c n^{2} \Gamma^{*}-c h v \Gamma+\frac{h \nu n(X+i Y)}{2 \pi(1+\chi)},
\end{aligned}
$$

and $B$ is a constant.

Also, results are in agreement with the work of El-Sirafy and Abdou [7].

(C) For $n_{1}=n_{2}=0,0<m<1$, and $\xi=(s+1) /(s-1)$, we get the mapping function $z=c\left(\xi+m \xi^{-1}\right)$. This function, in addition to functions (5.5), agrees with those of Muskhelishvili's obtained for the elliptic hole [9].

(D) For $m=-1$, the curvilinear holes degenerate into circular cuts.

(E) For $m=0,\left|n_{1}\right|<1$, and $\left|n_{2}\right|<1$, we get the mapping function

$$
z=c w(s)=c \frac{(s+1)^{3}}{(s-1)\left[(s+1)-n_{1}(s-1)\right]\left[(s+1)-n_{2}(s-1)\right]}, \quad n_{1} \neq n_{2} .
$$

Here the inner edges of the infinite plate are the inverse of elliptic limacon.

(F) Let $m=-n_{j}^{2}$. The hole is bounded by the circle $|z-n c|=c$ and the Goursal's functions are

$$
\begin{gathered}
k \phi(s)=A_{1}(s)+\frac{2 c \bar{\Gamma}}{1+s} \\
\psi(s)=A_{2}(s)+B_{1}(s)+\frac{2 c(\Gamma-k \bar{\Gamma})}{1+s} .
\end{gathered}
$$

6. Examples. (I) For $k=-1, \Gamma=p / 4, \Gamma^{*}=-(1 / 2) p e^{-2 i \theta}$, and $x=y=f=0$, the Goursat functions for our transformation take the form

$$
\begin{aligned}
\phi(s)= & \frac{p c}{1+s} \exp (2 i \theta)+\sum_{j=1}^{n} \frac{m+n_{j}^{2}}{\left(s+a_{j}\right)\left(1-n_{j}^{2}\right)}\left[p c-2 n_{j}^{2} J_{1 j} J_{2 j} L_{j}\right], \\
\psi(s)= & \frac{p c}{1+s}-p c \exp (-2 i \theta)+\sum_{j=1}^{2} \frac{m+n_{j}^{2}}{\left(s+a_{j}\right)\left(1-n_{j}^{2}\right)} \\
& +\frac{p \exp (2 i \theta)}{2(1+s)^{2}} \sum_{j=1}^{2}\left(1-n_{j}\right)^{2}\left(s+a_{j}+2\right) \\
& -\sum_{j=1}^{2} \frac{n_{j}^{2}\left(s+3 a_{j}\right)\left(m+n_{j}^{2}\right)}{\left(s+a_{j}\right)^{2}}\left[\frac{p c}{\left(1-n_{j}\right)^{2} J_{0 j}}+\frac{2 n_{j}^{2} J_{1 j} J_{2 j} L_{j}}{\left(1-n_{j}\right)^{2} J_{0 j}}\right],
\end{aligned}
$$


where

$$
\begin{aligned}
J_{1 j}= & {\left[n_{j}^{2}\left(m+n_{j}^{2}\right)-J_{0 j}\right]^{-1}, } \\
J_{2 j}= & {\left[-n_{j}^{2}\left(m+n_{j}^{2}\right)-J_{0 j}\right]^{-1}, } \\
L_{j}= & {\left[\left(1-n_{j}^{2}\right)^{2} \exp (-2 i \theta)-\left(m+n_{j}^{2}\right)\right] \frac{p c}{2} J_{0 j} } \\
& -\frac{c p n_{j}^{2}\left(m+n_{j}^{2}\right)}{2}\left[\left(m+n_{j}^{2}\right)-\left(1-n_{j}^{2}\right)^{2} \exp (2 i \theta)\right] .
\end{aligned}
$$

In the previous example, we have an infinite plate stretched at infinity by the application of a uniform tensile of intensity $p$, making an angle $\theta$ with the $\mathrm{x}$-axis. The plate is weakened by a curvilinear hole $c_{j}$, which is free from stress. If we put $n_{1}=n, n_{2}=0$ (or $n_{1}=0, n_{2}=n$ ) in (6.1), then the results agree with [5].

(II) For $k=-1, X=Y=\Gamma=\Gamma^{*}=0$, and $f=p t$, the complex functions $\phi(s)$ and $\psi(s)$ take the form

$$
\begin{aligned}
& \phi(s)=-2 c p \sum_{j=1}^{2} \frac{n_{j}\left(m+n_{j}^{2}\right)}{\left(1-n_{j}^{2}\right)\left(s+a_{j}\right)\left(n_{j}-n_{k}\right)}-\sum_{j=1}^{2} \frac{2 n_{j}^{2}\left(m+n_{j}^{2}\right) J_{1 j} J_{2 j} L_{j}}{\left(s+a_{j}\right)\left(1-n_{j}\right)^{2}}, \\
& \psi(s)=-\sum_{j=1}^{2} \frac{2 n_{j}^{4}\left(s+3 a_{j}\right)\left(m+n_{j}^{2}\right)^{2} J_{1 j} J_{2 j} L_{j}}{\left(1-n_{j}\right)^{2}\left(s+a_{j}\right)^{2} J_{0 j}} .
\end{aligned}
$$

The previous results give the solution of the first fundamental problem for an isotropic infinite plate with a curvilinear hole when there are no external forces and the edge of the hole is subject to a uniform pressure $p$. If $p=-i T$, we have the case when the edge of the hole is subject to a uniform tangential stress $T$.

(III) If $\Gamma=\Gamma^{*}=f=0$ and $k=\chi$, then the two complex functions are transformed to

$$
\begin{aligned}
& \phi(s)=\sum_{j=1}^{2} \frac{a_{j} n_{j}\left(m+n_{j}^{2}\right)\left(X J_{1 j}-i Y J_{2 j}\right) J_{0 j}}{\pi(1+\chi)\left(s+a_{j}\right)}, \\
& \psi(s)=\sum_{j=1}^{2} \frac{a_{j} n_{j}^{2}\left(s+3 a_{j}\right)\left(m+n_{j}^{2}\right)^{2}\left(X J_{1 j}-i Y J_{2 j}\right)}{\pi(1+\chi)\left(1+m n_{j}^{2}\right)\left(s+a_{j}\right)^{2}},
\end{aligned}
$$

where

$$
\begin{aligned}
J_{1 j} & =\left[\chi J_{0 j}+n_{j}^{2}\left(m+n_{j}^{2}\right)\right]^{-1}, \\
J_{2 j} & =\left[\chi J_{0 j}-n_{j}^{2}\left(m+n_{j}^{2}\right)\right]^{-1}, \\
L_{j} & =0 .
\end{aligned}
$$

Therefore, we have the solution of the second fundamental problem when a force $(x, y)$ acts on the curvilinear hole.

ACKNOwledgment. The author thanks prof. M. A. Abdou for his helpful discussions through this work. 


\section{REFERENCES}

[1] M. A. Abdou and S. A. Hassan, On the problem of stretched plates weakened by inner curvilinear holes, Banyan Mathematical Journal (India) 1 (1994), 245-253.

[2] M. A. Abdou and E. A. Khir Eldin, Stretched infinite plate weakened by an arbitrary curvilinear hole, Pure Appl. Math. 41 (1994), no. 2, 351-360.

[3] W. A. Bassali and R. H. Dawoud, Green's functions for thin isotropic plates containing holes, Proc. Cambridge Philos. Soc. 53 (1957), 755-763.

[4] W. R. Dean, Note on the Green's function of an elastic plate, Proc. Cambridge Philos. Soc. 50 (1954), 623-627.

[5] I. H. El-Sirafy, Stretched plates weakened by inner curvilinear holes, Z. Angew. Math. Phys. 28 (1977), no. 6, 1153-1159.

[6] _ On the problem of stretched infinite plate weakened by a hole having arbitrary shape, Indian J. Pure Appl. Math. 11 (1980), no. 1, 13-19.

[7] I. H. El-Sirafy and M. A. Abdou, First and second fundamental problems of infinite plate with a curvilinear hole, J. Math. Phys. Sci. 18 (1984), no. 2, 205-214.

[8] I. H. El-Sirafy, Some problems in the torsion of elastic beams and the bending of normally loaded thin elastic plates, Master's thesis, Faculty of Science, Alex. Univ., 1968.

[9] N. I. Muskhelishvili, Some basic problems of the mathematical theory of elasticity. Fundamental equations, plane theory of elasticity, torsion and bending, P. Noordhoff Ltd., Groningen, 1953.

I. S. Ismail: Department of Mathematics, Faculty of Science, Alexandria University, Egypt E-mail address: ismaai11958@hotmai 1.com 


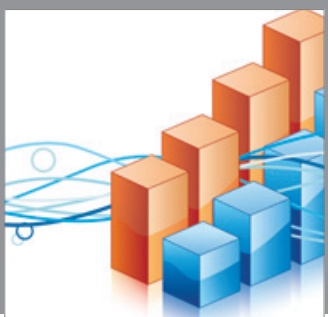

Advances in

Operations Research

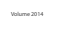

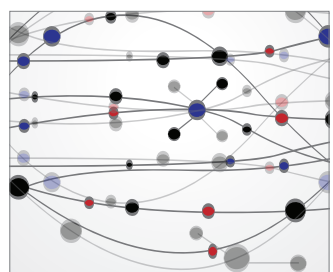

\section{The Scientific} World Journal
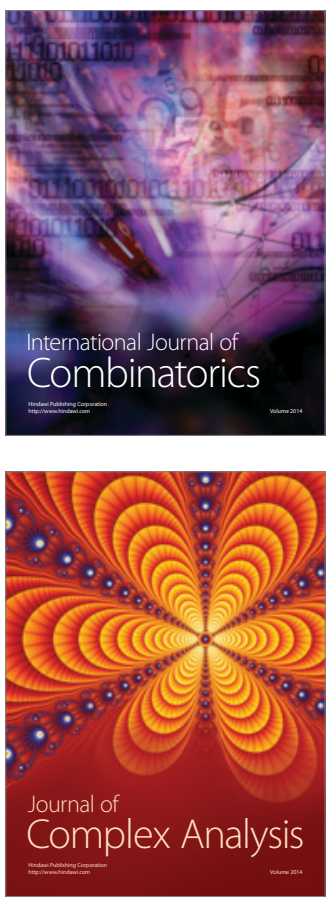

International Journal of

Mathematics and

Mathematical

Sciences
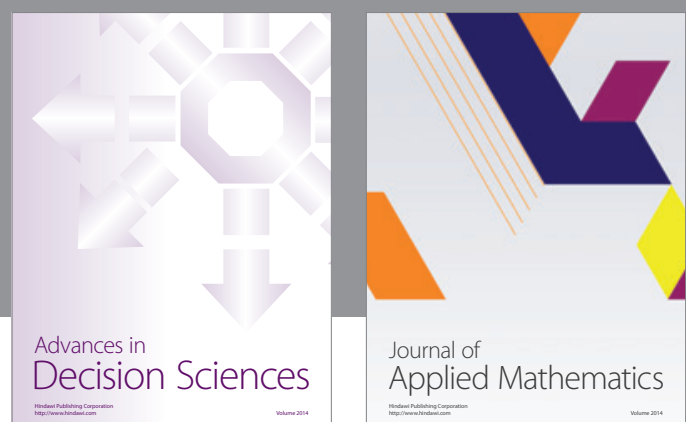

Journal of

Applied Mathematics
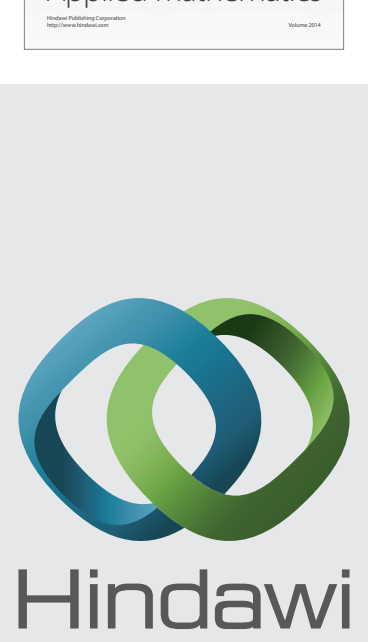

Submit your manuscripts at http://www.hindawi.com
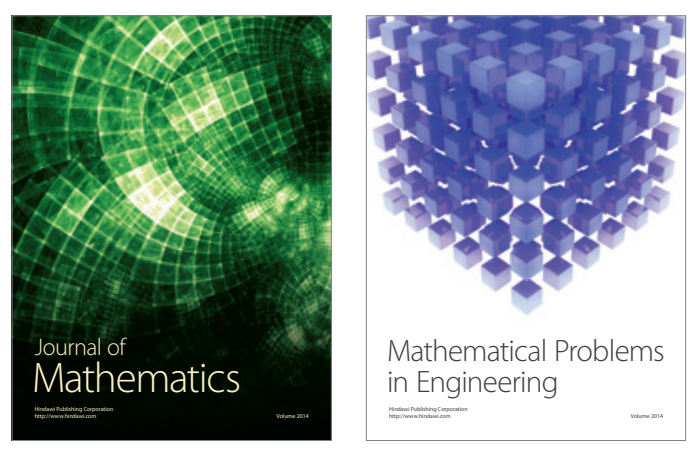

Mathematical Problems in Engineering
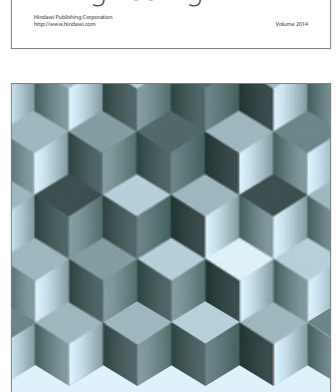

Journal of

Function Spaces
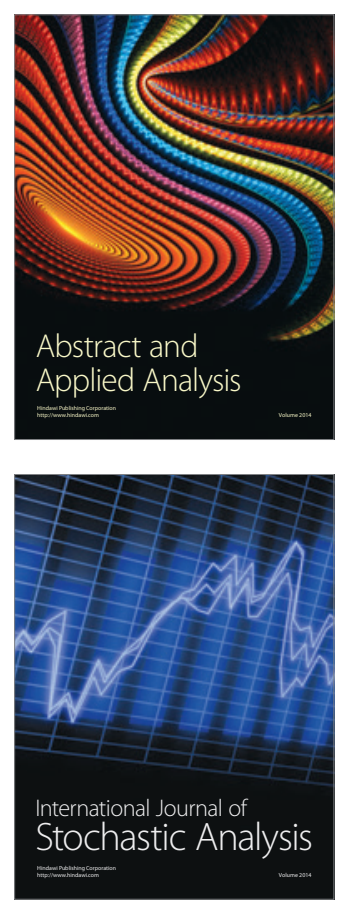

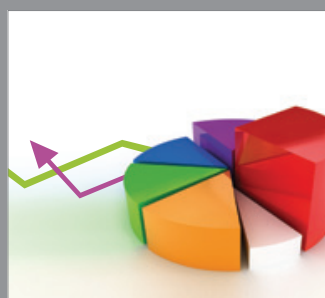

ournal of

Probability and Statistics

Promensencen
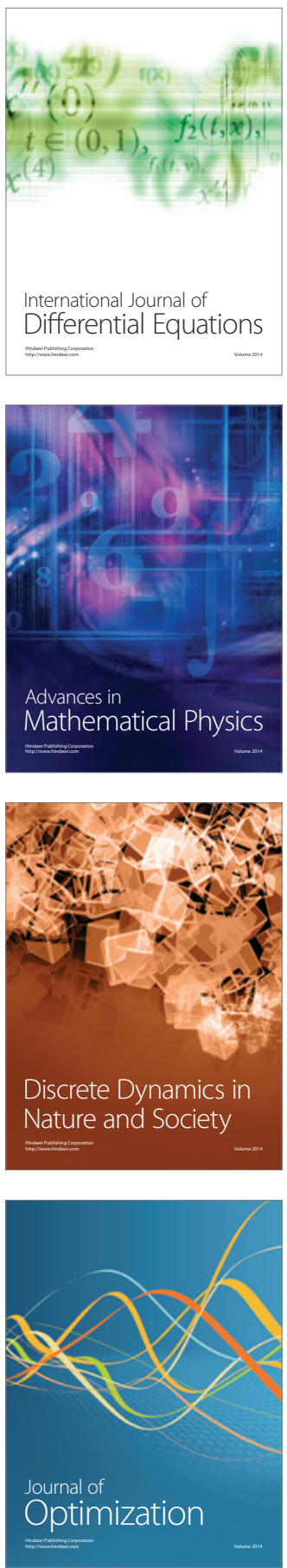